\title{
SIMULATION STUDIES AND BACKGROUND MEASUREMENTS FOR A LASER BASED BEAM SIZE MONITOR FOR THE FUTURE LINEAR COLLIDER
}

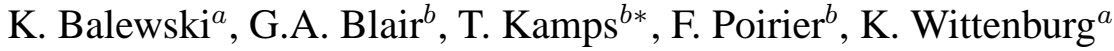 \\ ${ }^{a}$ Deutsches Elektron-Synchrotron DESY, D-22603 Hamburg, Germany \\ ${ }^{b}$ Royal Holloway University of London, Egham, Surrey, TW20 0EX, UK
}

\begin{abstract}
At several locations of the beam delivery system (BDS) of a future linear collider (FLC), beam spot sizes ranging from several hundreds to a few micrometers have to be measured. It is anticipated that laser wires will be used for this task in any FLC design. In order to optimize a laser wire system, simulations and background measurements have been carried out. Results are presented from simulations of the Compton scattering for the PETRA scenario. Furthermore results from measurements of backgrounds like synchrotron radiation and gas scattering have been measured at the positron storage ring PETRA at DESY and will be discussed.
\end{abstract}

\section{INTRODUCTION}

Laser wire scanners (LWS) will play an increasing role as the standard tool for beam size measurements [1] in the micrometer range for the BDS at any FLC design [2]. The LWS operation principle is based on the interaction between the electron beam and a finely focused laser beam. The electron bunch transverse size is measured by scanning the electron beam with the laser beam. Photons are then scattered out of the laser beam by Compton scattering. By counting the rate of Compton photons (or degraded electrons) as a function of the relative position of the laser waist and the electron beam, a measurement of the transverse beam size can be obtained. To specify the required laser beam power and detector performance, simulations and measurements of the backgrounds for such a measurement are of fundamental importance. In the following calculations of the signal level as well as of possible background sources are given. Furthermore results from background measurements in the relevant accelerator environment are discussed.

\section{COMPTON SCATTERING}

The fundamental process at work at the LWS is Compton scattering. In the classical limit with photon energies smaller than the electron energy and with electrons at rest, photon electron scattering is described by Thomson scattering. For high-energy photons non-classical effects must be taken into account leading to Compton scattering. With moving electrons the process is called inverse Compton

\footnotetext{
*t.kamps@rhul.ac.uk
}

scattering, where the moving electrons transfer energy to the photons yielding substantial fluxes of photons in the optical to X-ray region [3]. For the LWS, the total energy of the scattered photons per electron bunch laser pulse crossing is considered as the signal process. The number of photons $N_{C}$ is directly proportional to the laser beam power $P_{L}$ and wavelength $\lambda$ according to [4]

$$
N_{C}=N_{b} \frac{P_{L} \sigma_{C} \lambda}{c^{2} h} \frac{1}{\sqrt{2 \pi} \sigma_{s}} \exp \left(\frac{-y^{2}}{2 \sigma_{s}^{2}}\right)
$$

where $N_{b}$ is the number of electrons in a bunch, $y$ the relative offset between laser and electron beam and $\sigma_{s}^{2} \equiv$ $\sigma_{y}^{2}+\omega_{o}^{2}$ the overlap region. The electron beam size is $\sigma_{y}$ and the laser beam waist at the interaction point is $\omega_{o}$. The Compton cross section $\sigma_{C}$ is in Fig. 1 evaluated for two scenarios: One for a typical linear collider test facility
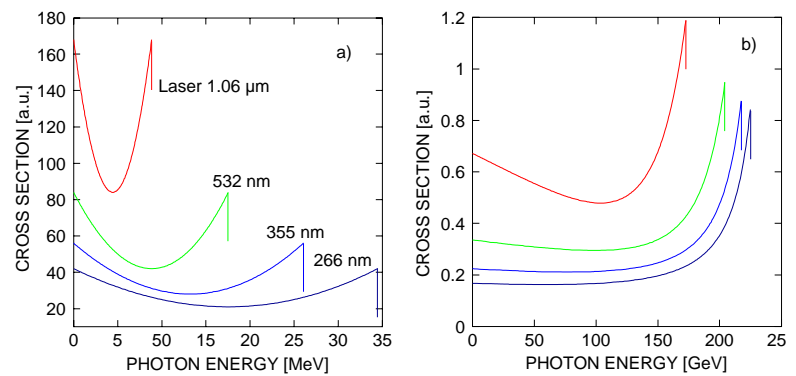

Figure 1: Compton cross section for the first four harmonics of an Nd:YAG laser scanning a $1 \mathrm{GeV}$ (a)and $250 \mathrm{GeV}$ (b) electron beam.

beam energy $(1 \mathrm{GeV})$, where sub-systems of a LWS will be tested and for a typical linear collider beam delivery system energy $(250 \mathrm{GeV})$. Currently a Nd:YAG based laser system is the instrument of choice because of its performance capabilities with respect to high power and small spot size.

\section{BACKGROUND SOURCES}

For the use of a LWS, the background conditions at the detector locations are important. The biggest source of background photons in the low energy region is synchrotron radiation emitted by the electron beam in bending magnets. The spectrum of synchrotron radiation is characterized by the critical energy which is in the $\mathrm{keV}$ region for beam energies of several GeV. Another background source arises from beam gas scattering. Here the electrons 
in the beam interact with the residual gas. Bremsstrahlung is the dominant process at high energies with a cross section combined from the individual cross sections from photon emission at the nucleus and emission at the bound electron. The bremsstrahlung spectrum for emitted photons with energy $E_{\gamma}$ goes up to the beam energy $E_{b}$ proportional to $\left(E_{\gamma} / E_{b}\right)^{-1}$. Both spectra, for synchrotron radiation and bremsstrahlung are plotted in Fig. 2.
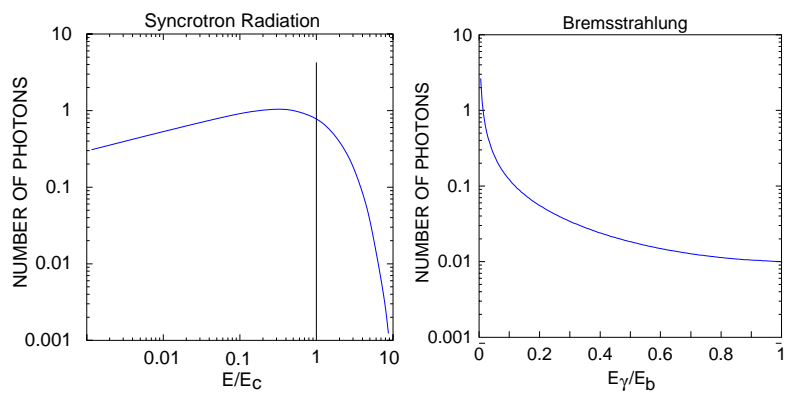

Figure 2: Photon spectrum from synchrotron radiation and bremsstrahlung.

\section{SIMULATIONS}

The aim of the simulation studies is a full Monte Carlo simulation of the signal (Compton) and all relevant background processes. This includes the modeling of the complete measurement setup, from the scattering process to the analog-digital-converter (ADC) of the readout electronics of the detector. This is of fundamental importance for the specification of the laser system and the detector. So far the Compton process is modeled in a realistic accelerator environment including beampipe, magnets, and vacuum windows. The parameter set for the electron beam are for the PETRA positron storage ring at DESY in Hamburg, because prototype tests of a LWS are foreseen with this machine. The simulation work is carried out in the Geant4 [5] framework. The standard toolkit is used for multiple scattering while the low energy electromagnetic toolkit [6] is includes Compton and Rayleigh scattering, photoelectric effect, bremsstrahlung, and ionization at energies $250 \mathrm{eV}$. A specific Monte Carlo generator [7] is implemented for synchrotron radiation photons. In Fig. 3 simulation results are shown for the Compton process with PETRA accelerator parameters. The low energy peak in the spectrum is caused by multiple scattering processes at low energy in the $2 \mathrm{~mm}$ steel vacuum window. Full Monte Carlo simulations of the background processes are under way.

\section{BACKGROUND MEASUREMENTS}

Preliminary background measurements have been performed at the positron storage ring PETRA. Two locations were used for the measurements at two different energies. In Fig. 4 the measurement setup is depicted. A Cs(I) crystal $\left(1.5 \times 1.5 \times 10 \mathrm{~cm}^{3}\right)$ mounted to a photomultiplier was
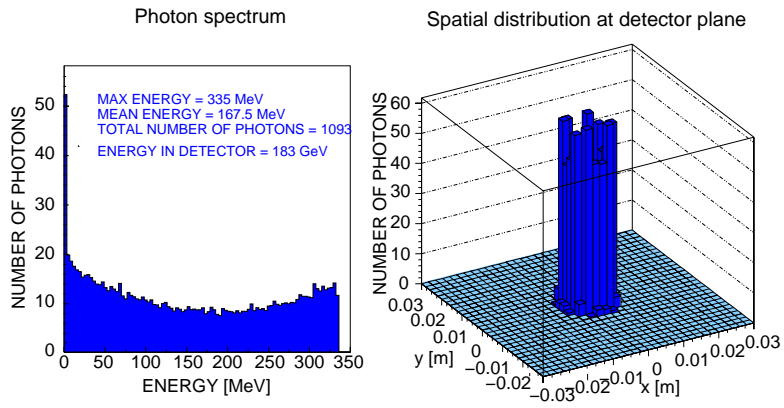

Figure 3: Photon spectrum of Compton photons simulated with Geant4. Scattering of a $10 \mathrm{MW}$ peak power green $(\lambda=532 \mathrm{~nm})$ laser beam with $1 \mu \mathrm{m}$ spot size at a $4.5 \mathrm{GeV}$ electron beam with transverse beam size of $100 \mu \mathrm{m}$. For the spatial distribution calculation the detector positioned $4 \mathrm{~m}$ away from the interaction.

used. The crystal and the photomultiplier are packed together with a support structure in a light tight box made of lead. An aperture is drilled on the side facing the electron beam. The whole package is taped with black tape and positioned at the two locations tangent to the beampipe and $30 \mathrm{~cm}$ away from the dipole magnets to avoid any electromagnetic effects on the detector. Before making the measurements the detector together with its readout electronics were calibrated in terms of energy using a radioactive source. The beam parameters of the positron beam at PETRA relevant for the measurements are gathered in Tab. 1 Results from measurements at the two locations with two

$\begin{array}{lcc}\text { Beam energy } & 4.5 \text { and } 7 & \mathrm{GeV} \\ \text { Beam current } & 1.55 \text { to } 1.77 & \mathrm{~mA} \\ \text { Particles per bunch } & 7.5 \text { to } 8.5 & 10^{10} \\ \text { Repetition rate } & 130 & \mathrm{kHz} \\ \text { Bending angle } & 28.08 & \mathrm{mrad} \\ \text { Vacuum pressure } & 1 \text { and } 2 & 10^{-10} \mathrm{mbar}\end{array}$

Table 1: PETRA beam parameters relevant for background measurements.

beam energies are plotted in Fig. 5. Synchrotron radiation and bremsstrahlung are the two dominant background sources. The energy of the photons from synchrotron radiation at $4.5 \mathrm{GeV}$ is too low $\left(E_{C} \simeq 1 \mathrm{keV}\right)$ to pass the beampipe material. At $7 \mathrm{GeV}$ the background is a superposition of synchrotron radiation with strong components of bremsstrahlung. At location two, after the long straight section, the electrons have to pass through about 16 times more residual gas, which increases the rate of bremsstrahlung by that amount.

\section{CONCLUSIONS}

Laser wire scanners (LWS) will play an increasing role as the standard tool for beam size measurements at future linear collider. To fully exploit the potential of such a de- 


\section{Proceedings of the 2001 Particle Accelerator Conference, Chicago}

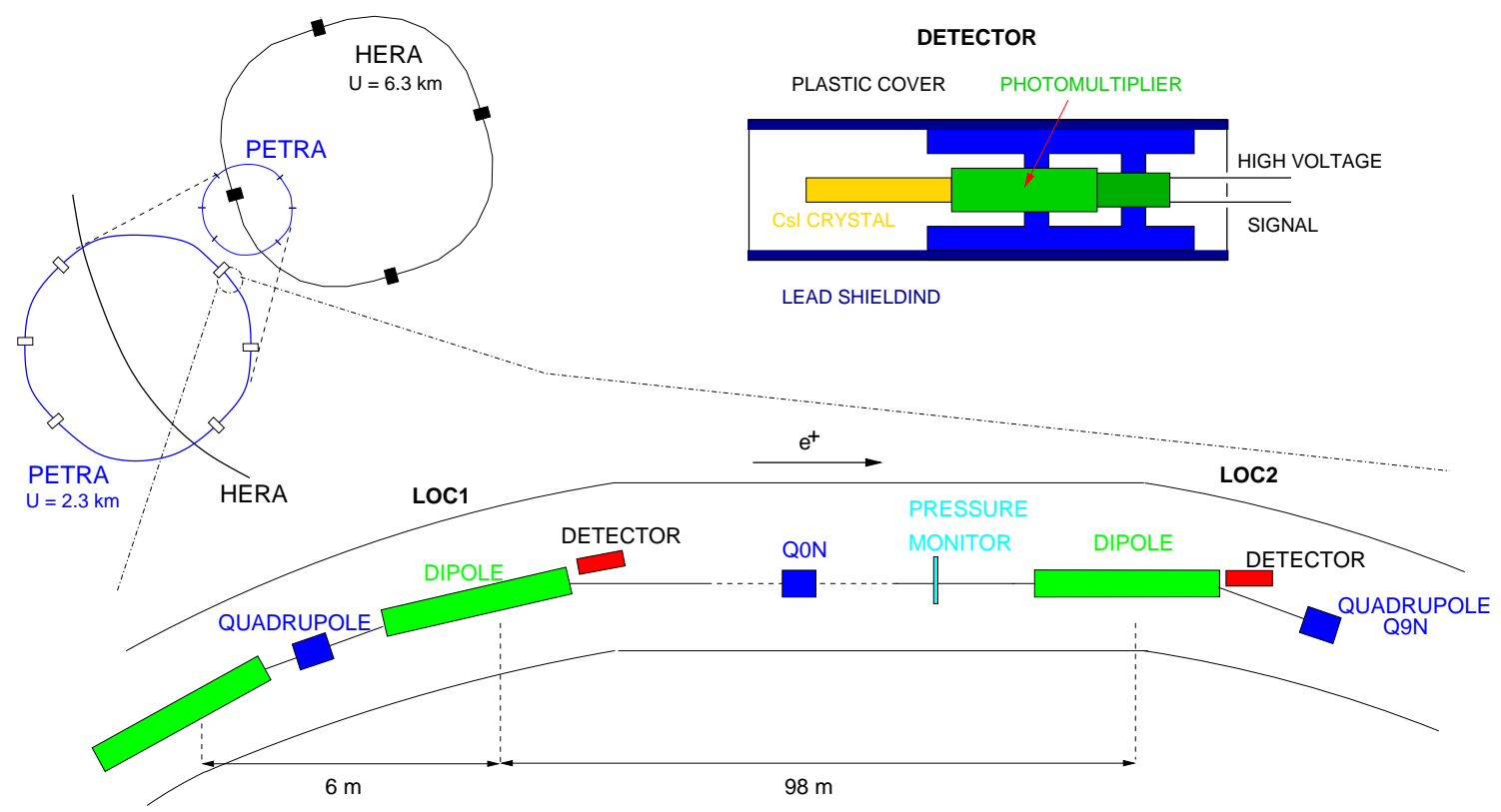

Figure 4: Schematic setup for background measurements at PETRA.
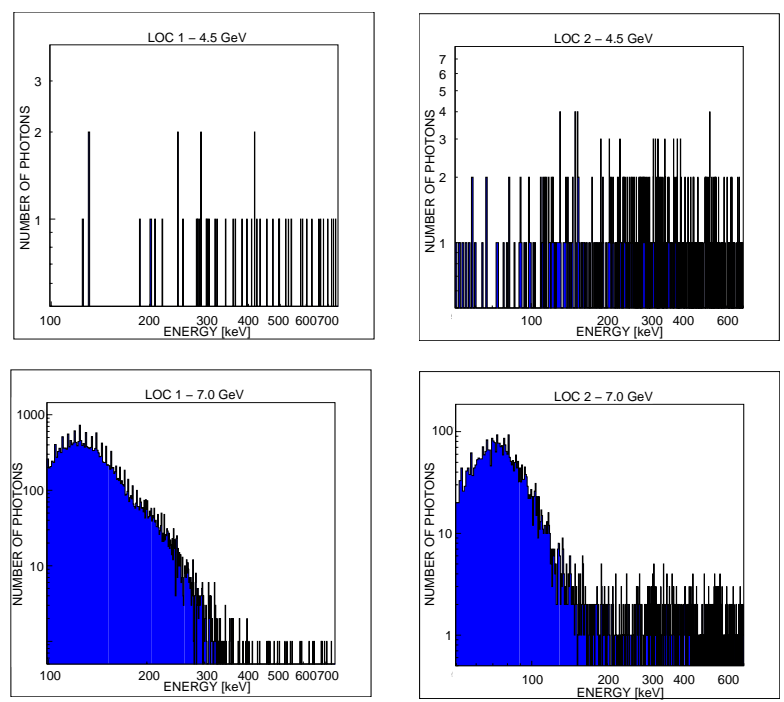

Figure 5: Background spectra measured at PETRA. Data was taken over a $1 \mathrm{sec}$ long period for each measurement.

vice, studies are under way to supply full Monte Carlo simulations of all relevant processes connected with the LWS. Furthermore background measurements at the PETRA accelerator have been performed, enabling laser and detector specification for a system test of a LWS. A much faster detector, made of lead tungstate, is under study. It might enable a $10 \mathrm{MHz}$ sampling rate which is the nominal bunch rate at PETRA.

\section{REFERENCES}

[1] G. A. Blair, J. Frisch, K. Honkavaara, T. Kamps, F. Poirier, I. N. Ross, M. Ross, H. Schlarb, P. Schmueser, S. Schreiber,
D. Sertore, N. Walker, M. Wendt, K. Wittenburg "Proposing a Laser Based Beam Size Monitor for the Future Linear Collider," PAC 2001, Chicago, TPAH051, this proceedings

[2] The various worldwide Linear Collider workshop proceedings and documentation of ongoing activities can be accessed from the central website: http://lcwws.physics.yale.edu/lc/

[3] I. F. Ginzburg, G. L. Kotkin, V. G. Serbo and V. I. Telnov, "Colliding Gamma E And Gamma Gamma Beams From Single Pass Electron Positron Accelerators," Sov. J. Nucl. Phys. 38 (1983) 222

[4] T. Shintake, "Proposal of nanometer beam size monitor for e+ e- linear colliders," Nucl. Instrum. Meth. A 311 (1992) 453.

[5] K. Amako [Geant4 Collaboration], "Present status of Geant4,” Nucl. Instrum. Meth. A 453 (2000) 455.

[6] J. Apostolakis, S. Giani, M. G. Pia, L. Urban, M. Maire and P. Nieminen, "GEANT4 low energy electromagnetic models for electrons and photons," CERN-OPEN-99-034.

[7] H. Burkhardt, "Monte Carlo Generator For Synchrotron Radiation,” CERN-LEP-NOTE-632. 